IRA-International Journal of Management \& Social Sciences

ISSN 2455-2267; Vol.06, Issue 03 (2017)

Pg. no. 440-460

Institute of Research Advances

https://research-advances.org/index.php/RAJMSS

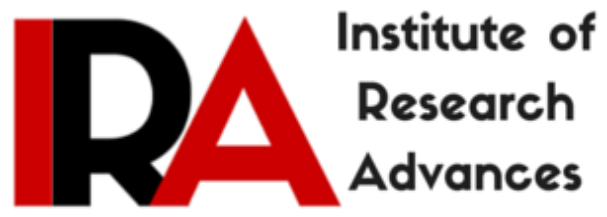

\title{
Developing Actuarial Assumptions within the Framework of a Hyper-Inflationary Environment
}

\author{
Evaristo Diz Cruz (Ph.D.) ${ }^{1}$ \\ President, EDiz Actuarial Services and Consulting \\ Academic Director, Universidad Católica Andrés Bello, Venezuela \\ Santa Paula Professional Center Tower B, 8th Floor, Ofic. 803 \\ Av. Circunvalación del Sol, Urb. Holy Paula \\ El Cafetal, Caracas 1061-A, Venezuela.
}

J. Tim Query (Ph.D., C.P.A., A.R.M.) ${ }^{2 \#}$

Professor \& Mountain States Insurance Group Endowed Chair

New Mexico State University, MSC 3FIN, P.O. Box 30001

Las Cruces, NM 88003, U.S.A.

\#corresponding author

Type of Review: Peer Reviewed.

DOI: http://dx.doi.org/10.21013/jmss.v6.n3.p10

\section{How to cite this paper:}

Cruz, E., \& Query, J. (2017). Developing Actuarial Assumptions within the Framework of a Hyper-Inflationary Environment. IRA-International Journal of Management \& Social Sciences (ISSN 2455-2267), 6(3), 440-460. doi:http://dx.doi.org/10.21013/jmss.v6.n3.p10

(C) Institute of Research Advances

(cc) EY-NC

This work is licensed under a Creative Commons Attribution-Non Commercial 4.0 International License subject to proper citation to the publication source of the work.

Disclaimer: The scholarly papers as reviewed and published by the Institute of Research Advances (IRA) are the views and opinions of their respective authors and are not the views or opinions of the IRA. The IRA disclaims of any harm or loss caused due to the published content to any party. 


\begin{abstract}
The primary objective of this research is to determine the impact of actuarial assumptions for work liability valuations in Venezuela. The time frame of interest to the study finds that Venezuela has and continues to experience an extremely volatile rate of inflation. In such an environment, it is imperative that the discount rate be set as a function of the inflation rate plus a premium. This study is focused on the challenges of adopting such a policy given the absence of a well-developed capital market.
\end{abstract}

Keywords: Actuarial assumptions payments; multivariate linear Regression Retroactivity; Models; Statistical analysis; distribution curves fits; multivariate regression; Time series; Curve and distributions fitting; Actuarial assumptions; set of curves and distributions; multivariable regression models; actuarial liabilities; IAS 19.

JEL Codes: C65, G23, J64.

\title{
Introduction
}

One of the major concerns when making actuarial assumptions is the choice of rates of inflation. These rates are generally provided through such factors as the macroeconomics of the country; the performance of sovereign bonds with high credit quality used to determine interest rates for discounting the obligations; and rates of wage increases as defined by corporate compensation policies with the framework of the economic environment under which companies operate.

In developed and mature countries these variables are relatively clearly defined through economic stability and low volatility, which allows for relatively accurate and steady forecasts, with low prediction errors. Obviously, current conditions in much of South America and especially in Venezuela are not conducive to precise forecasting. The purpose of this study analyzes the relationships between these variables to determine the equanimity of actuarial assumptions as required by the International Accounting Standards, specifically IAS $19 .{ }^{1}$ Using practical examples to illustrate the modeling of liabilities under different scenarios, this research defines the respective impact of each of the assumptions used, based on the empirical relationship between inflation and interest rates.

The current economic environment in Venezuela is a dire one. Venezuela's consumer inflation, already the world's highest, is projected to more than double this year to a level above all estimates from economists surveyed by Bloomberg, according to the International Monetary Fund.

\footnotetext{
${ }^{1}$ IAS19 or International Accounting Standard Nineteen is an accounting rule concerning employee benefits under the IFRS rules set by the International Accounting Standards Board. In this case, "employee benefits" includes wages and salaries as well as pensions, life insurance, and other perquisites.
} 


\section{Graph 1 - Inflation in Venezuela 2006-2015}

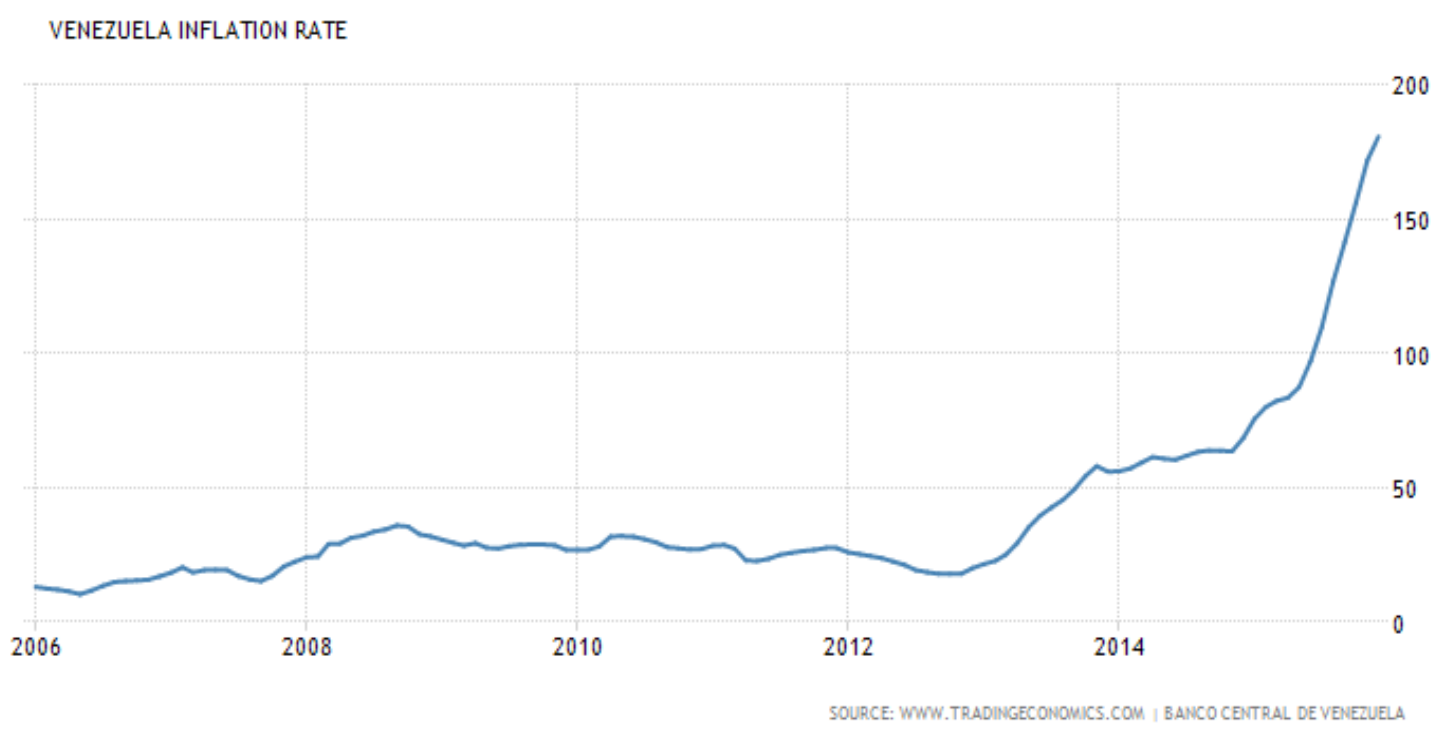

According to a note published by the IMF's Western Hemisphere Director, Alejandro Werner, Inflation will surge to 720 percent in 2016 from 275 percent last year. For comparison, that percentage is nearly quadruple the median 184 percent estimate from 12 economists surveyed by Bloomberg, and exceeding the highest forecast of 700 percent from Nomura Securities. Spiking prices and widespread shortages for even staples have driven discontent in Venezuela, and helped the opposition gain control of Congress for the first time in a decade as President Nicolas Maduro attempts to turn the tide of what he has deemed an "economic emergency." (Bloomberg, 2016)

Table 1

Estimated Venezuela Inflation, end of period consumer prices, percent change

\begin{tabular}{|c|c|c|c|c|c|}
\hline $\mathbf{2 0 1 6}$ & $\mathbf{2 0 1 7}$ & $\mathbf{2 0 1 8}$ & $\mathbf{2 0 1 9}$ & $\mathbf{2 0 2 0}$ & $\mathbf{2 0 2 1}$ \\
\hline $720 \%$ & $2,200 \%$ & $3,000 \%$ & $3,600 \%$ & $4,100 \%$ & $4,600 \%$ \\
\hline
\end{tabular}

Source: International Monetary Fund

\section{Theoretical Considerations}

Following the logic of the Capital Asset Pricing Model (CAPM) for pricing capital assets, we quickly identified the key variables impacting the discount rate. In some respects, we see the discount rate as a function of the capital markets, eventually constructing a portfolio consisting of stocks and bonds that define a specific performance measure that can be referenced when determining the discount rate. Defining the most important relationships between the inflation rate, the rate of wage increases, and the interest rate used to discount future obligations is important.

In the worlds of finance and actuarial science, the formula used to determine the current value and/or actuarial $V_{0}$, of a defined benefit plan with a duration of $n$ years. 


$$
V_{0}=-A+\sum_{k=1}^{n} \frac{Q_{k}}{\prod_{h=1}^{k}\left(1+i_{r}\right)}+\frac{V_{n}}{\prod_{h=1}^{n}\left(1+i_{r}\right)},
$$

$V_{0}:$ Actual Value

\begin{tabular}{|l|}
\hline \hline Ir: Interest Rate \\
\hline$Q k:$ Flow payments of death benefits \\
\hline
\end{tabular}

$V_{n}:$ Flow payments of final benefits if it survives

The previous formula can easily be assimilated to a certain payment $Q_{k}$ if an employee leaves in each one of the $k$ moments for a particular contingency and $V_{n}$ if it survives the previous and arrives at the time $n$.

In this formula we consider that all elements are stochastic:

\section{The Cash Flows}

\section{The Duration}

\section{The Types of Interest}

If the cash flows are random, they could be replaced by their equivalent certain and the expression above would thus be:

$$
V_{0}=\sum_{k=1}^{n} \frac{E\left(Q_{k}\right)}{\prod_{h=1}^{k}\left(1+i_{r}\right)}+\frac{E\left(V_{n}\right)}{\prod_{h=1}^{n}\left(1+i_{r}\right)} ; V R=\sum_{k=1}^{n} \frac{E\left(R_{k}\right)}{\prod_{h=1}^{k}\left(1+i_{r}\right)}
$$

However, all of the types of interest referred to above represent the most significant challenge to the evaluator as he or she resolves to determine the actuarial value of a plan. This is due to small variations that can cause large variations in the current value and/or actuarial assumptions. If the chosen rates are high, there will be an under-estimation. On the contrary, if the rates used are too low, an overvaluation will result. It is expected that analysts with aversion to risk will choose higher interest rates and those who are closer to being risk seekers will opt for smaller types of interest rates. Therefore, it is in this determination where the greatest amount of subjectivity likely resides within this form of evaluation.

The level of interest rates will depend to some degree on both the general economic conditions in that country and the specific situation estimated for the individual company. When a company's own individual prospects (unsystematic risk) are unfavorable, higher interest rates are used to capture the added risk. Updating interest rates using the sum of a base level (among the various options, the most appropriate is to consider the interest rate associated with long-term operations with no risk due to the 
creditworthiness of the debtor. This is represented by the legal rate of money, the types of long-term public debt, etc.), plus a risk premium (including economic risk, financial risk, and liquidity risk). In such a case:

$$
\mathrm{i}_{\mathrm{m}}=\mathrm{i}+\mathrm{P}_{\mathrm{i}}+\mathrm{sp} \text { Being: }
$$

$\mathrm{i}_{\mathrm{m}}$ : Interest to discount

1. The base type of interest without risk

$\mathrm{P}_{\mathrm{i}}:$ Interest for premium

$\mathrm{SP}$ : Spread of credit

Obviously, the above is true in countries where the risk-free rate is somewhat reliably determinable. However, in the case of Venezuela during the time frame of interest to this study, that has certainly not been the case.

We believe that another version with the same structure, but based on inflation as $\boldsymbol{i}_{m}=I+\Delta_{\text {may }}$ be helpful in formulating a usable discount rate. The rate of inflation in Venezuela in recent years has been quite high, particularly in the year 2015. Inflation recognized by the Banco Central de Venezuela (BCV) was in the order of $189 \%$ on an annualized basis. So in theory, the first component alone would already an interest rate higher than the rate in nominal terms.

Being:

\section{$\boldsymbol{i}_{m:}$ Interest rate to discount}

$$
i_{m}=I+\Delta
$$

\section{I: Inflation rate}

\section{$\triangle:$ Additional Premium}

Future forecasted refresh rate (interest rates) can be performed through an extrapolation of past values, though, using such methodologies as a regression analysis and/or models of stochastic time series. The traditional approach, as analyzed so far, considers the types of interest deterministically, i.e. deliberates on the only possible value for the same factor in each period. However, given that the future is not known, it will be more reasonable to create several possible scenarios, which will then be reduced to a single best fit with the help of statistical treatments (Cruz and Valls, 2002a). A more correct and rational approach is to use the mathematical expectation of the different values, since the mathematical expectation of the different values is the value for which the set of all possible deviations is minimal. Basically, it is a question of considering the updated rate of each year as a random variable.

From a mathematical point of view, a problem arose with a special emphasis on the difficulty of the algorithms leading to the determination of the average discount factor, known the distribution function of the random variable that describes the rate of interest applicable to a period. In the next section we 
derive from the data ${ }^{2}$ historical monthly inflation measures as well as the performance of the National Public Debt Bonds utilizing the empirical probability distributions based on the sample evidence of the respective time series.

\section{Descriptive Comparison of the Time Series (Inflation and Performance in Venezuela)}

In Venezuela when comparing the changing levels of monthly inflation with the average monthly yields of sovereign bonds of the national public debt (Global Bonds) we find the following:

\section{Graph 2}

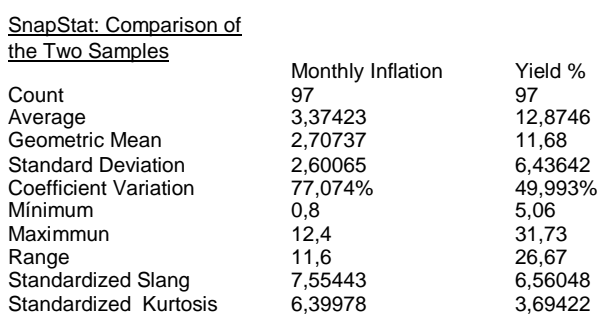

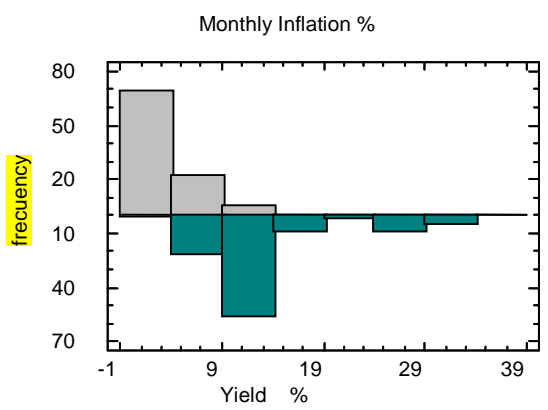

Chart of Box and Whiskers

Confidence Interval of $95 \%$

Dif. of Means : $-9,50041+/--1,39482 \quad[-8,10559,-10,8952]$

Variance Reason: $[0,109166,0,244153]$

\section{Comparison of Means}

Null Hypothesis : difference $=0$

Statistical $=-13,4786$ Value-P Billateral $=0,0000$

Monthly Inflation \%

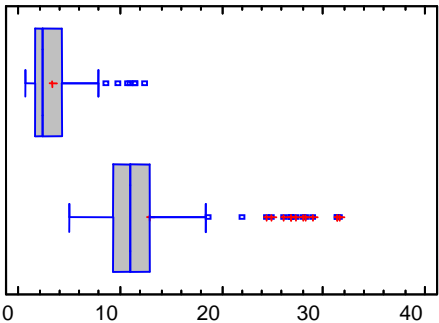

Comparison of Sigmas

Statistical $F=0,163258$ Value-P Billateral $=0,0000$

Yield \%

Diagnostics

Values-P Shapiro-Wilks $=0,0000$ y 0,0000

Autocorrelation in Delay $1=0,9037+/-0,1990,0,9176+/-0,1990$
A
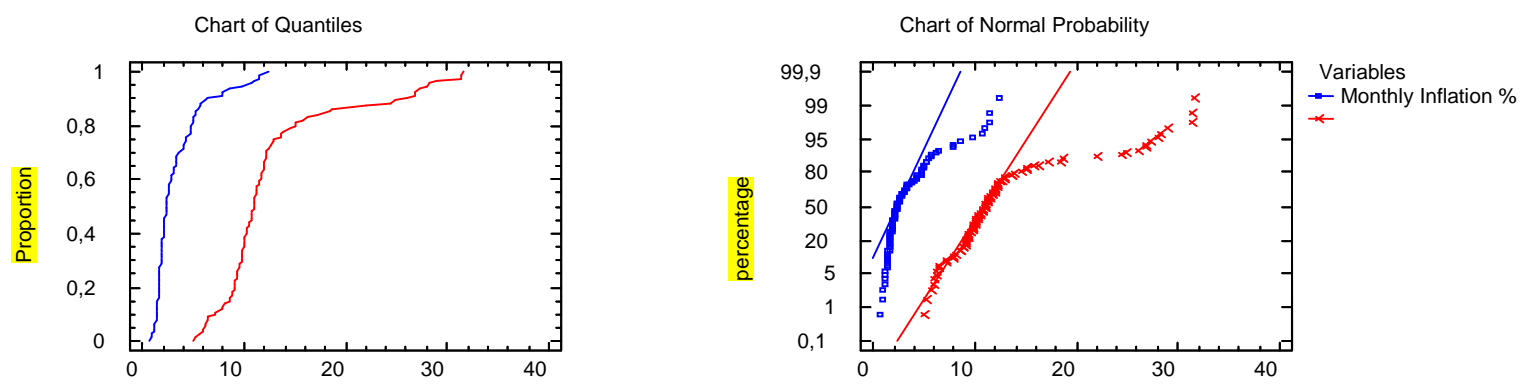

${ }^{2}$ Monthly data from 2007 until 2015 published by EMBI Venezuela and the Central Bank of Venezuela (BCV). 
In the probability graph it can be clearly seen that both performance and inflation are far from exhibiting normal behavior. Greater volatility can also be seen in the levels of monthly inflation, as viewed in terms of the coefficient of variation. The arithmetic and geometric averages, respectively of the coefficient of the yields of the series are: - 2.707373 .37423 for inflation and $12.8746-11.68$ for the yields.The foregoing supports the hypothesis that yields are higher on average than the levels of inflation. Based on this supposition it seems reasonable to use in principle a discount rate that could be constructed as the sum of the inflation and an additional measure.

\section{Modeling of the Inflation Rate and Yields}

Our analysis includes the evaluation of 15 different models of probability distributions. By comparing probability models our purpose is to identify those models that are best adapted to reflect true inflation rates and yields. Our results follow:

Table 2 - Comparison of alternative distributions

\begin{tabular}{|l|c|c|c|c|c|}
\hline \multicolumn{1}{|c|}{ Distribution } & Est. Parameter & $\begin{array}{c}\text { Realistic } \\
\text { Log }\end{array}$ & Chi-Square P & KS D & $\boldsymbol{A}^{\wedge 2}$ \\
\hline $\begin{array}{l}\text { Log logistic (3- } \\
\text { Parameters) }\end{array}$ & 3 & $\mathbf{- 1 8 5 , 5 1 5}$ & 0,00923487 & 0,066567 & 0,59672 \\
\hline \multicolumn{1}{|c|}{ Gaussian Reverse } & 2 & $-188,224$ & 0,0153393 & 0,121158 & 1,57656 \\
\hline Birnbaum-Saunders & 2 & $-189,251$ & 0,0291259 & 0,130922 & 1,87101 \\
\hline Lognormal & 2 & $-189,579$ & 0,0102683 & 0,117006 & 1,60955 \\
\hline Log logistic & 2 & $-190,886$ & 0,00288731 & 0,086189 & 1,45403 \\
\hline Gamma & 2 & $-197,891$ & 0,000109807 & 0,15565 & 3,1488 \\
\hline The most extreme value & 2 & $-202,129$ & $9,31457 \mathrm{E}-05$ & 0,162588 & 3,82363 \\
\hline Weibull & 2 & $-203,416$ & $6,68831 \mathrm{E}-05$ & 0,151661 & 3,73823 \\
\hline Exponential & 1 & $-214,968$ & $9,84 \mathrm{E}-13$ & 0,257126 & 7,49062 \\
\hline Laplace & 2 & $-214,998$ & $2,65 \mathrm{E}-09$ & 0,200507 & 6,15745 \\
\hline Logistic & 2 & $-221,249$ & $1,68 \mathrm{E}-07$ & 0,162651 & 5,31173 \\
\hline Normal & 2 & $-229,846$ & $1,90 \mathrm{E}-13$ & 0,210529 & 7,57786 \\
\hline Uniform & 2 & $-237,747$ & 0 & 0,475204 & \\
\hline The smallest value & 1 & $-259,271$ & 0 & 0,266987 & 11,5107 \\
\hline Pareto & & $-1,00 \mathrm{E}+09$ & $6,24 \mathrm{E}-07$ & 0,221546 & \\
\hline
\end{tabular}

\subsection{Adjustment of Data not Censored - Monthly Inflation}

Data/Variable: monthly inflation \%

97 values ranging from 0.8 to 12.4 
Table 3 - Adjusted distributions

\begin{tabular}{|c|c|c|c|}
\hline $\begin{array}{c}\text { Gaussian } \\
\text { Reverse }\end{array}$ & Log Logistic & $\begin{array}{c}\text { Log logistic } \\
\text { (3Parameters) }\end{array}$ & Normal \\
\hline Medium $=3,37423$ & Medium=2,57051 & Medium $=1,73786$ & Mean = 3.37423 \\
\hline Scale= 2,07247 & Form=03239 & Form $=0,528573$ & $\begin{array}{c}\text { Standard Deviation }= \\
2,60065\end{array}$ \\
\hline & & $\begin{array}{c}\text { Lower threshold= } \\
0,734177\end{array}$ & \\
\hline
\end{tabular}

In accordance with the statistical log likelihood, the distribution of best fit is the distribution log logistics of 3 parameters, which is characterized by the three parameters of distributions that are displayed in the table above.

The probability density function of inflation is given by:

\section{Graph 3 - Probability Density Function}

\section{CHART OF THE SMOOTH DENSITY FOR}

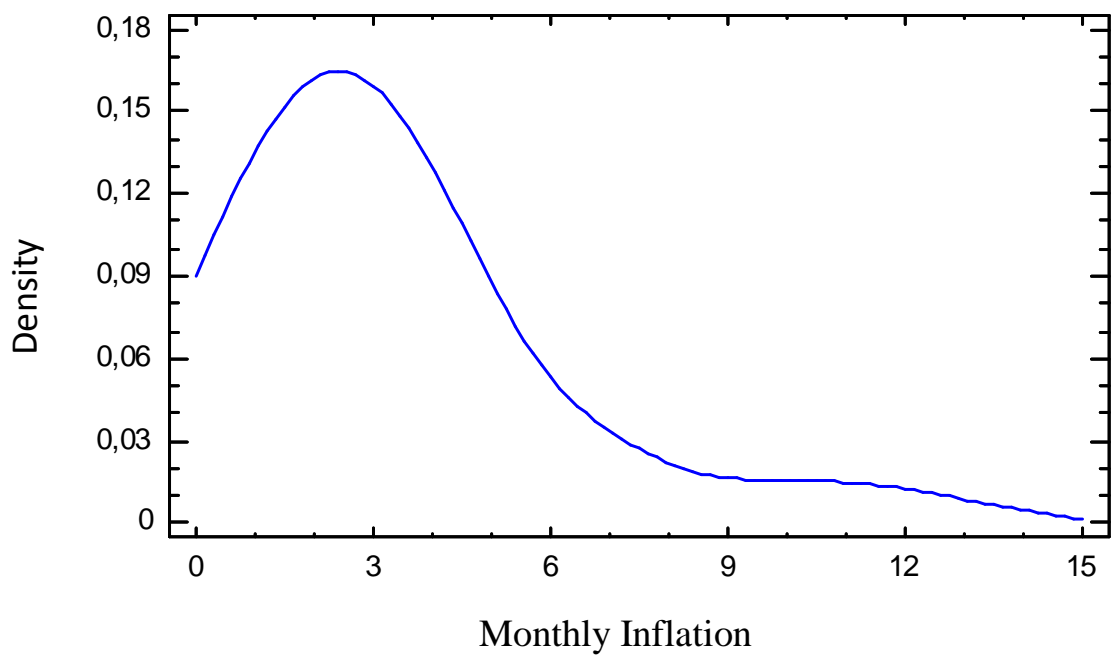

The process similarly repeats after adjusting the data to a probability distribution of yields. 


\subsection{Adjustment of data not censored - Yield \%}

Data/Variable: Performance\%

97 values ranging from 5.06 to 31.73

Table 4 - Adjusted distributions

\begin{tabular}{|c|c|c|c|}
\hline Loglogistic & Loglogistic (3Parameters) & Lognormal & Normal \\
\hline medium = 11.2417 & medium $=7.20247$ & mean $=12.7764$ & mean =12.8746 \\
\hline form = 0.22672 & form $=0.352999$ & $\begin{array}{c}\text { standard deviation } \\
\mathbf{5 . 6 6 4 2 6}\end{array}$ & $\begin{array}{c}\text { standard deviation } \\
=6.43642\end{array}$ \\
\hline & lower threshold $=3.88637$ & $\begin{array}{c}\text { Scale log: } \text { media }= \\
\mathbf{2 . 4 5 7 8 8}\end{array}$ & \\
& & $\begin{array}{c}\text { log scale } \text { est. } \text { dev }= \\
0.423605\end{array}$ & \\
\hline
\end{tabular}

P-values lower than 0.05 would indicate that the performance\% does not come from the selected distribution with $95 \%$ confidence.

In both cases we conclude that both distributions are not normal of the analysis the distributions of best fit are logistics tri-parametrics who have maximum likelihood and the lowest value using the KolmogorovSmirnorv test.

\section{Adjustment of Autoregressive Integrated Moving Average (ARIMA) Model for Short-term and Medium-term Forecasting}

The above statement obviously is based on the historical information regarding the monthly performance of inflation from 2007 to 2015. However, we do not have any guarantee that these trends remain in the future. This requires us to make projections of at least short and medium-term future scenarios in order to quantify in some way if this differential was maintained on average. Below are the most noteworthy results of the autoregressive models of comprehensive moving averages with annual seasonal adjustments ARIMA type.

\section{Summary of ARIMA Model for the Rates of Return and Inflation}

The modeled periods are months based on the history of both variables. On the basis of the forecasted averages of each, we can establish the differences between the two rates. 
Table 5 - ARIMA Models

Yield Summary

\begin{tabular}{|l|l|l|l|l|}
\hline Parameter & Estimated & $\begin{array}{c}\text { Estimated } \\
\text { Error }\end{array}$ & T & P-Value \\
\hline AR(1) & 0,258713 & 0,104689 & 2,4713 & $\mathbf{0 . 0 1 5 3 3}$ \\
\hline SAR(1) & 0,898247 & 0,196205 & 4,5781 & $1,5 \mathrm{E}-05$ \\
\hline SAR(2) & 0,557164 & 0,32266 & 1,7268 & 0,0876 \\
\hline SMA(1) & 0,589241 & 0,30282 & 4,5228 & $1,9 \mathrm{E}-05$ \\
\hline SMA(2) & 0,937789 & 0,251492 & 3,7289 & 0,00033 \\
\hline
\end{tabular}

Inflation summary

\begin{tabular}{|l|l|l|l|c|}
\hline Parameter & Estimated & $\begin{array}{c}\text { Estimated } \\
\text { Error }\end{array}$ & T & P-Value \\
\hline AR(1) & 0,719815 & 0,149729 & 4,8075 & $\mathbf{6 E - 0 6}$ \\
\hline AR(2) & $-0,317099$ & 0,110374 & $-2,8725$ & 0,00507 \\
\hline MA(1) & 0,736021 & 0,127716 & 5.763 & 0 \\
\hline SAR(1) & 1,32992 & 0,0233672 & 56,914 & 0 \\
\hline SMA(1) & 1,30475 & 0,0367575 & 35,496 & 0 \\
\hline
\end{tabular}

\begin{tabular}{|c|c|c|c|}
\hline \multicolumn{5}{|c|}{ Predicted Yield \% } \\
\hline Period & Data & Prediction & Residual \\
\hline $1 / 50$ & 5,06 & & \\
\hline $2 / 50$ & 5,14 & 5,69548 & $-0,555483$ \\
\hline $3 / 50$ & 5,62 & 5,01737 & 0,602627 \\
\hline $4 / 50$ & 6,38 & 5,84672 & 0,533283 \\
\hline $5 / 50$ & 6,23 & 6,55507 & $-0,325065$ \\
\hline $6 / 50$ & 5,86 & 6,57913 & $-0,719127$ \\
\hline $7 / 50$ & 5,91 & 5,73713 & 0,172866 \\
\hline $8 / 50$ & 6,38 & 5,95677 & 0,423232 \\
\hline $9 / 50$ & 6,26 & 6,6681 & $-0,408098$ \\
\hline $10 / 50$ & 9,3 & 6,6886 & 2,6114 \\
\hline $11 / 50$ & 14,65 & 10,6021 & 4,04793 \\
\hline $12 / 50$ & 15,07 & 16,0974 & $-1,02742$ \\
\hline $1 / 51$ & 18,62 & 16,0581 & 2,56189 \\
\hline $2 / 51$ & 17,37 & 19,6848 & $-2,31481$ \\
\hline $3 / 51$ & 16,16 & 16,7094 & $-0,549358$ \\
\hline $4 / 51$ & 15,67 & 16,151 & $-0,480978$ \\
\hline $5 / 51$ & 12,94 & 14,85 & $-1,90999$ \\
\hline $6 / 51$ & 13,02 & 12,4088 & 0,611179 \\
\hline $7 / 51$ & 11,86 & 13,0719 & $-1,21189$ \\
\hline $8 / 51$ & 11,17 & 11,7488 & $-0,578805$ \\
\hline
\end{tabular}

\begin{tabular}{|c|c|c|c|}
\hline \multicolumn{4}{|c|}{ Predicted Inflation $\%$} \\
\hline Period & Data & Forecast & Residual \\
\hline $1 / 50$ & 3,1 & & \\
\hline $2 / 50$ & 3,1 & 2,91844 & 0,181561 \\
\hline $3 / 50$ & 2,1 & 2,59104 & $-0,491045$ \\
\hline $4 / 50$ & 1,7 & 2,0237 & $-0,323698$ \\
\hline $5 / 50$ & 1,7 & 1,93489 & $-0,234888$ \\
\hline $6 / 50$ & 3,2 & 1,98994 & 1,21006 \\
\hline $7 / 50$ & 2,4 & 3,27524 & $-0,875243$ \\
\hline $8 / 50$ & 1,9 & 1,98299 & $-0,0829859$ \\
\hline $9 / 50$ & 1,8 & 1,86319 & $-0,0631865$ \\
\hline $10 / 50$ & 2 & 1,89942 & 0,100585 \\
\hline $11 / 50$ & 2,4 & 2,09254 & 0,307456 \\
\hline $12 / 50$ & 2,3 & 2,31528 & $-0,0152787$ \\
\hline $1 / 51$ & 2,6 & 1,86378 & 0,736224 \\
\hline $2 / 51$ & 2,3 & 2,6151 & $-0,315102$ \\
\hline $3 / 51$ & 1,3 & 1,61569 & $-0,315692$ \\
\hline $4 / 51$ & 1,2 & 1,30422 & $-0,104224$ \\
\hline $5 / 51$ & 1,8 & 1,49909 & 0,300912 \\
\hline $6 / 51$ & 2 & 2,08435 & $-0,0843454$ \\
\hline $7 / 51$ & 1,8 & 1,84039 & $-0,0403872$ \\
\hline $8 / 51$ & 2,1 & 1,64399 & 0,45601 \\
\hline
\end{tabular}




\begin{tabular}{|c|c|}
\hline \multicolumn{2}{|c|}{ Differentials(R-I) } \\
\hline Period & Differential \\
\hline $2 / 50$ & 2,78 \\
\hline $3 / 50$ & 2,43 \\
\hline $4 / 50$ & 3,82 \\
\hline $5 / 50$ & 4,62 \\
\hline $6 / 50$ & 4,59 \\
\hline $7 / 50$ & 2,46 \\
\hline $8 / 50$ & 3,97 \\
\hline $9 / 50$ & 4,80 \\
\hline $10 / 50$ & 4,79 \\
\hline $11 / 50$ & 8,51 \\
\hline $12 / 50$ & 13,78 \\
\hline $1 / 51$ & 14,19 \\
\hline $2 / 51$ & 17,07 \\
\hline $3 / 51$ & 15,09 \\
\hline $4 / 51$ & 14,85 \\
\hline $5 / 51$ & 13,35 \\
\hline $6 / 51$ & 10,32 \\
\hline $7 / 51$ & 11,23 \\
\hline $8 / 51$ & 10,10 \\
\hline
\end{tabular}

Differential average: $8.57 \%$

It is logically intuitive to conclude that with a greater time projected, there is greater uncertainty and the differential tends to grow.

\section{A Numerical Application to a Small Venezuelan Company}

Given that the empirical evidence supports the thesis affirming the interest rate for discounting; it can be expressed as $\boldsymbol{i}_{m}=I+\Delta$, at least in Venezuela.

Being:

im: Interest rate to discount

\section{I: Inflation rate}

$\triangle:$ Additional Premium

Using this information, we develop various scenarios with different assumptions and settings to see the impact of each one in terms of Projected Benefit Obligation (PBO) or actuarial liability to the valuation date. It is important to mention at this stage of the work that the new scenarios would maintain a 4 per cent spread when betting on an economic recovery of the country in the medium and long term.

\section{A. General and Actuarial Assumptions}

In this section we manage two types of actuarial assumptions - one based on nominal rates and the other based on real rates. In the case of the two scenarios with nominal rates, we observe two different versions: the first one uses fixed rates for both the discount rate as the wage increase and using the same average wage increases from the table of wages in STAGE I; the second version uses variable rates for the increase of wages. The other groups of scenarios are based on actual rates varying in both the discount rate and wage growth rate. 


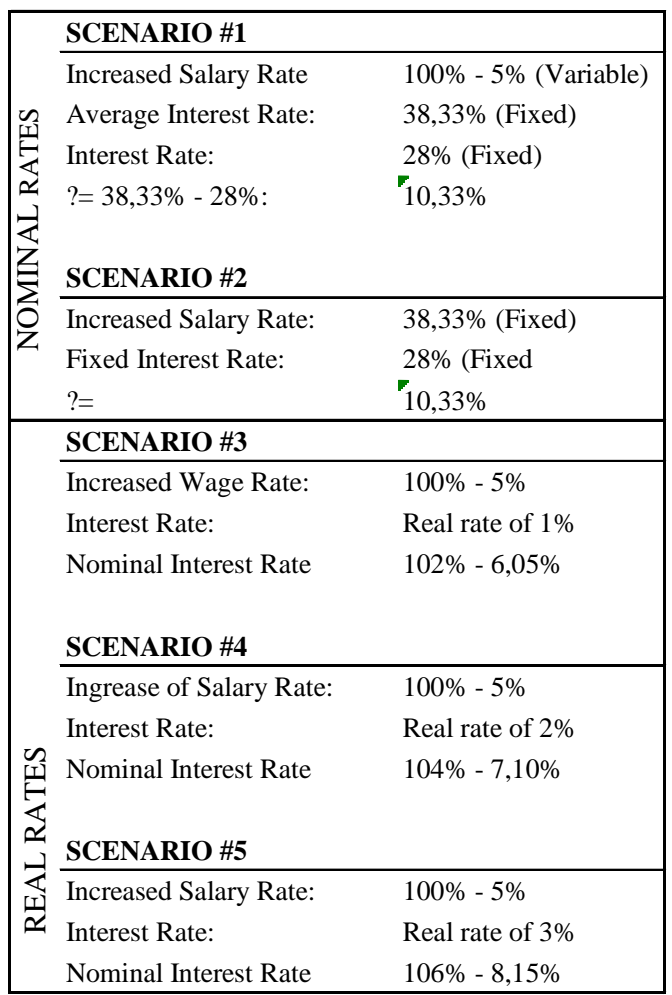

\begin{tabular}{|c|r|r|r|r|}
\cline { 2 - 5 } \multicolumn{1}{c|}{} & \multicolumn{3}{c|}{ Real Rate } & \multicolumn{1}{c}{} \\
\cline { 2 - 5 } \multicolumn{1}{c|}{} & $\mathbf{1 \%}$ & $\mathbf{2 \%}$ & $\mathbf{3 \%}$ & \multicolumn{1}{c|}{} \\
\hline Salary & \multicolumn{3}{c|}{ Interest Rate } & Years \\
\hline $100,00 \%$ & $102,00 \%$ & $104,00 \%$ & $106,00 \%$ & 2016 \\
\hline $80,00 \%$ & $81,80 \%$ & $83,60 \%$ & $85,40 \%$ & 2017 \\
\hline $60,00 \%$ & $61,60 \%$ & $63,20 \%$ & $64,80 \%$ & 2018 \\
\hline $40,00 \%$ & $41,40 \%$ & $42,80 \%$ & $44,20 \%$ & 2019 \\
\hline $30,00 \%$ & $31,30 \%$ & $32,60 \%$ & $33,90 \%$ & 2020 \\
\hline $20,00 \%$ & $21,20 \%$ & $22,40 \%$ & $23,60 \%$ & 2021 \\
\hline $10,00 \%$ & $11,10 \%$ & $12,20 \%$ & $13,30 \%$ & 2022 \\
\hline $5,00 \%$ & $6,05 \%$ & $7,10 \%$ & $8,15 \%$ & 2023 \\
\hline $5,00 \%$ & $6,05 \%$ & $7,10 \%$ & $8,15 \%$ & 2024 \\
\hline
\end{tabular}

Table 6 - Demographic data of the company

\begin{tabular}{|l|r|r|r|}
\hline \multicolumn{1}{|c|}{ CONCEPT } & \multicolumn{1}{|c|}{ FEM } & \multicolumn{1}{|c|}{ MALE } & \multicolumn{1}{c|}{ TOTAL } \\
\hline Population & 59 & 81 & 140 \\
Current Age (Average) & 33,59 & 39,40 & 36,95 \\
Current Service (Average) & 3,94 & 3,81 & 3,87 \\
Integral Salary (Average) (Bs.) & $56.349,12$ & $75.052,77$ & $67.170,52$ \\
Payroll / Month (Bs.) & $3.324 .597,93$ & $6.079 .274,41$ & $9.403 .872,34$ \\
\hline Guarantee PS in x (Bs.) & $11.218 .798,09$ & $15.286 .999,55$ & $26.505 .797,64$ \\
Retroactive PS in x (Bs.) & $20.419 .511,62$ & $23.133 .605,84$ & $43.553 .117,45$ \\
Payable PS in x (Bs.) & $20.874 .035,20$ & $24.306 .427,04$ & $45.180 .462,24$ \\
Payable Dif vs Guarantee in x (Bs.) & $9.655 .237,11$ & $9.019 .427,49$ & $18.674 .664,60$ \\
\hline
\end{tabular}

\section{B. Summary of the main results in terms of the actuarial liability}

The following results were obtained using the above assumptions. 
Table 7 - Actuarial Liabilities

Scenarios Retroactive Liability PBO Differential Service Cost Interest Cost Nominal Rates Real Rates

\begin{tabular}{|c|l|l|l|l|c|}
\hline Scenarios & $\begin{array}{l}\text { Retroactive } \\
\text { Liability }\end{array}$ & $\begin{array}{l}\text { PBO } \\
\text { Differential }\end{array}$ & Service Cost & Interest Cost & $\begin{array}{c}\text { Rates } \\
\text { Nominal/Real }\end{array}$ \\
\hline I & 79.36 & 58.91 & $7.530 .084,29$ & $15.095,605,24$ & Nominal Rates \\
\cline { 1 - 4 } II & 99.43 & 73.67 & $13.653 .453,83$ & $20.629 .543,11$ & \\
\hline III & 40.36 & 15.76 & $1.767 .161,86$ & $16.081 .529,02$ & \multirow{2}{*}{ Real Rates } \\
\cline { 1 - 4 } IV & 37.61 & 18.43 & $1.448 .502,83$ & $13.968 .403,93$ & \\
\cline { 1 - 4 } V & 35.23 & 12.44 & $1.185 .851,40$ & $12.131 .106,85$ & \\
\hline
\end{tabular}

Table 7 above infers that all scenarios could coexist depending on which assumptions are used. Obviously there are two quite distinct trends by the order of magnitude of the results:

i. The first trend is based on nominal rates (the first two scenarios) which tend to generate an actuarial liability by the same order of magnitude but obviously greater than that generated using real rates.

ii. The second trend is based on the use of real rates which involves adjusting for inflation the nominal rates of wages and of the discount. This second approach leads to liabilities that are estimated on the low end of the order of magnitude (particularly since we tend to take on most of the actuarial valuations) given the levels of inflation observed in the country. This is supported by empirical evidence that yields exceed the rates of inflation, and it appears that the use of actual rates would support this practice.

If we assume that the wage growth rates can be expressed as a function of inflation, which is true for the vast majority of the wage policies of Venezuelan companies, then it is possible to express the interest rate as a function also of wages as follows:

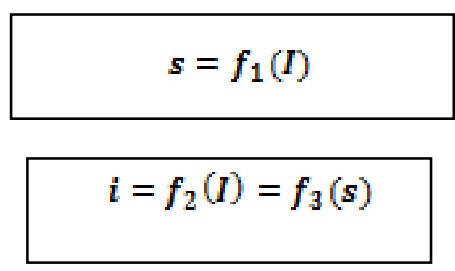

Being:

s: Rate of increase wage

$i$ : Interest rate

I: inflation rate

$r$ : Interest real rate to discount

Following Fisher, it is then possible to posit that given a wage policy that reflects the inflation-we shall call it $f_{a}(s)$, then, $(1+s)(1+r)=(1+i)$ would make economic sense for all future years, due to the differential of the historical rates between inflation and yields. As you can see in the graph they are in the order of $8.57 \%$, and when you adjust for future autoregressive models of the ARIMA type, conditioned by the historical data, the average rate differential is positive. It seems reasonable then to use 
a real rate of up to 4 percentage points on the wage increase rate for discounting the obligations. While it is noteworthy to observe that in the future the differential appears stable, it is also important to note that in a scenario of an improving national economy, it may be possible to maintain this trend with declining wage rates.

Assuming that the theoretical differential between the retroactivity and the guarantee to the valuation date is in the order of $18.67 \mathrm{MMBs}$, in deterministic terms it seems reasonable to use real interest rates that cover the liabilities accurately that is $100 \%$ or more of projected liabilities for future differentials of the residue between the PBO differential and the differential of the current certain nonprojected liabilities.

\section{Conclusion}

In this study we attempt to determine the impact of actuarial assumptions for work liability valuations in Venezuela. The time frame of interest to the study makes it unique, in that the South American country of Venezuela has and continues to experience an extremely volatile rate of inflation. Our research focuses on the challenges of adopting such a policy given the absence of a well-developed capital market.

The difference when using real rates is that it recognizes the cost of inflation in the period in which it occurs increasing obviously the cost of interest of next year to recognize such as interest rate inflation + another measure, minimizing as much as possible the actuarial losses that usually occur in economies as volatile as the current Venezuelan economy.

In the appendices we provide in detail all germane calculations developed in the preparation of this work. It is important to mention that our methodology assumes future scenarios rates with annual declines are treated by maintaining a positive spread in all scenarios, and are obviously betting on a general improvement of the economy.

\section{References}

1. Biller, David, (2016) “IMF Sees Venezuela Inflation Rocketing to 720 Percent in 2016,”January 22, 2016 http://www.bloomberg.com/news/articles/2016-01-22/imf-sees-venezuela-inflation-rocketing-to-720percent-in-2016.

2. Dey, A. K., \& Kundu, D. (2009). Discriminating between the log-normal and log-logistic distributions. Communications in Statistics-Theory and Methods, 39(2), 280-292.

3. Pérez-Cruz, Fernando, Gustavo Camps-Valls, Emilio Soria-Olivas, Juan José Pérez-Ruixo, Aníbal R. Figueiras-Vidal, and Antonio Artés-Rodríguez, (2002) "Multi-dimensional Function Approximation and Regression Estimation," Artificial Neural Networks - ICANN 2002,Volume 2415 of the series Lecture Notes in Computer Science, pp 757-762. 


\section{Appendices}

\section{Forecasts of Future Performance \%}

Data/Variable: Performance \%

Number of observations $=97$

Initial index $=1 / 50$

Sample interval $=1,0$ month $(\mathrm{s})$

Length of the seasonality $=12$

\section{Summary of Forecasts}

Non-seasonal differentiation of order: 1

Forecasting Model Selected: ARIMA $(1,1,0)$ x $(2,0,2) 12$

Number of forecasts generated: 96

Number of periods retained for validation: 0

\begin{tabular}{|l|l|l|}
\hline Statistical & $\begin{array}{l}\text { Period of } \\
\text { Estimate }\end{array}$ & $\begin{array}{l}\text { Period of } \\
\text { Validation }\end{array}$ \\
\hline RMSE & 1,62507 & \\
\hline MAE & 1,22786 & \\
\hline MAPE & 9,57007 & \\
\hline I & $-0,0467702$ & \\
\hline MPE & $-0,675507$ & \\
\hline
\end{tabular}

\section{Summary of ARIMA Model}

\begin{tabular}{|l|l|l|l|l|}
\hline Parameter & Dear & Std error. & $T$ & $P$-Value \\
\hline AR(1) & 0,258713 & 0,104689 & 2,47126 & 0,015325 \\
\hline SAR(1) & 0,898247 & 0,196205 & 4,57811 & 0,000015 \\
\hline SAR(2) & 0,557164 & 0,32266 & 1,72678 & 0,087600 \\
\hline SMA(1) & 0,589241 & 0,130282 & 4,5228 & 0,000019 \\
\hline SMA(2) & 0,937789 & 0,251492 & 3,72891 & 0,000334 \\
\hline
\end{tabular}




\section{Historical Forecast: Yes}

Estimated variance of white noise $=2,77929$ with 91 degrees of freedom

Estimated standard deviation of white noise $=1,66712$

Number of Iterations: 17

This procedure predicts future values of the performance percentage. The data cover 97 periods of time. Currently, we have selected the model of an autoregressive integrated moving average (ARIMA). This model assumes that the best prognosis available for future data is given by the parametric model that relates the most recent value with the prior values and previous noise. Each performance value $\%$ has been adjusted in the following way, before adjusting the model:

(1) We take simple differences of order 1.

The output summarizes the statistical significance of the terms in the model forecasts. Terms with Pvalues lower than 0.05 were statistically different from zero with a confidence level of $95.0 \%$. The Pvalue for the term $\mathrm{AR}(1)$ is less than 0.05 , so that is statistically different from 0 . The P-value for the term SAR(2) is greater than or equal to 0.05 , so that is not statistically significant. Therefore, should consider reducing the order of the term SAR to 1. The P-value for the term SMA(2) is less than 0.05, so that is statistically different from 0 . The estimated standard deviation of the white noise of input is equal to 1,66712 .

This table also summarizes the performance of the model currently selected in adjust historical data. Shown:

(1) the root mean square error (RMSE)

(2) the mean absolute error (MAE)

(3) the percentage of mean absolute error (MAPE)

(4) the average error (I)

(5) the percentage of average error (MPE)

Each one of the statistics based on the forecast errors are one-forward, which are the differences between the data at time $t$ and the forecasted value at time $t-1$. The first three statistics measure the magnitude of the errors. The smaller this value, the more robust the model. The last two statistics measure the bias. Similarly, a better model would provide a value closest to 0 . 


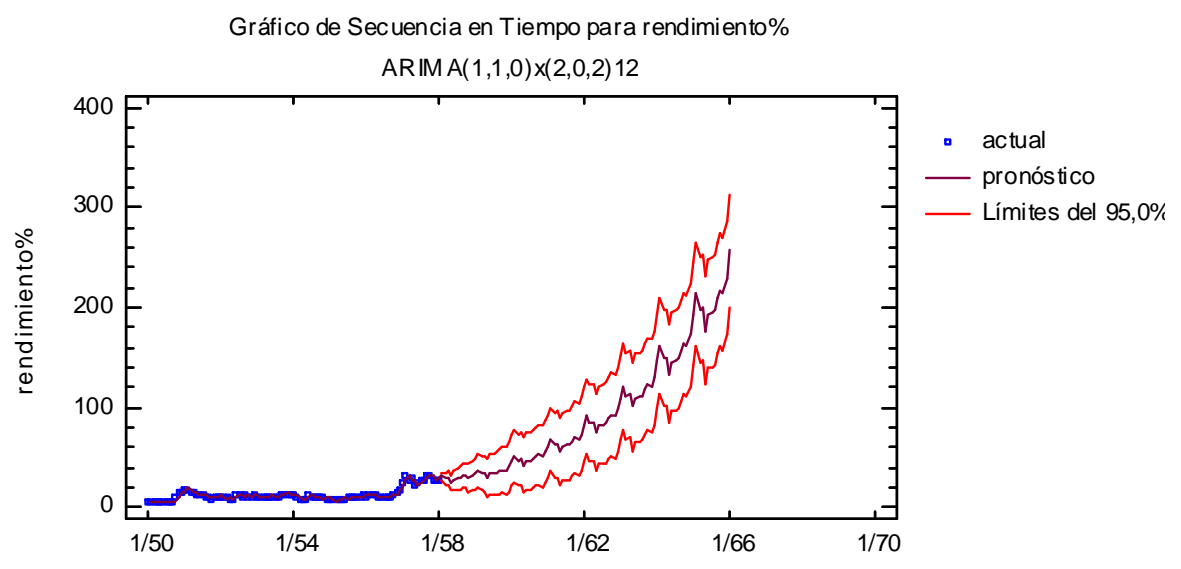

Table of forecasts for performance \%

Model: ARIMA $(1,1,0) \times(2,0,2) 12$

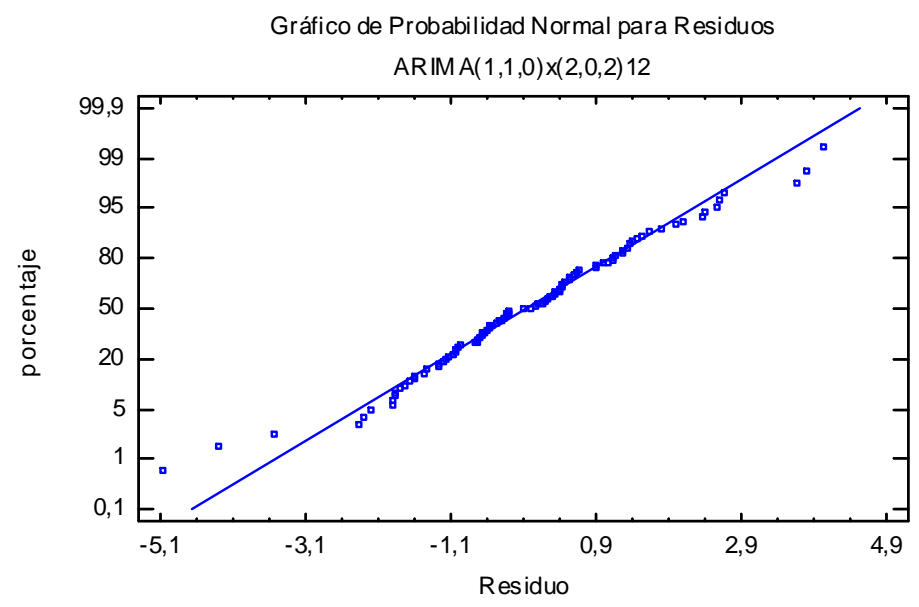




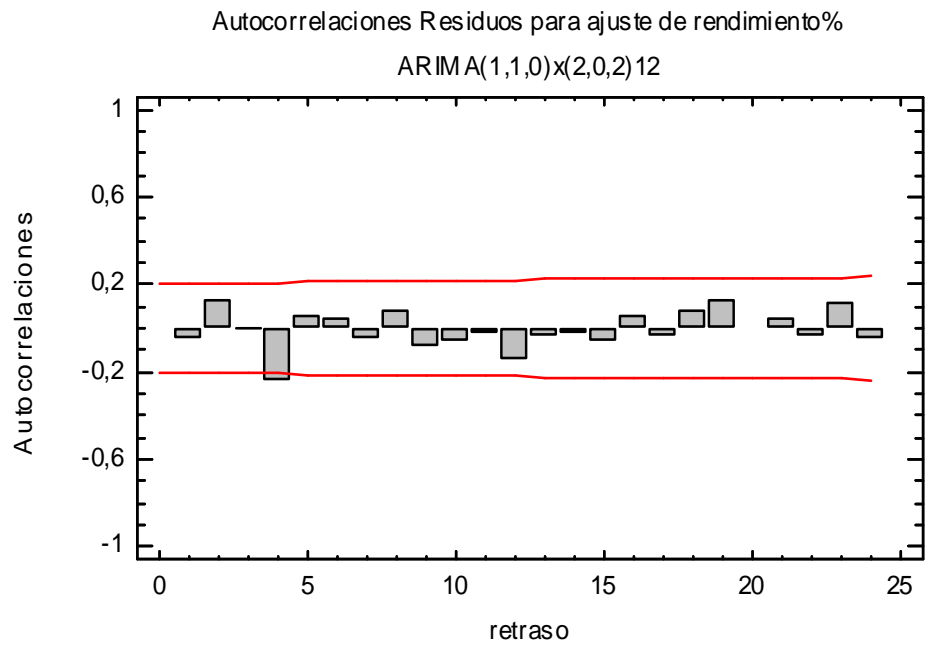

\section{II . Automatic forecasts - Monthly Inflation \%}

Data/Variable: monthly inflation \%

Number of observations $=97$

Initial index $=1 / 50$

Sample interval $=1,0$ month $(\mathrm{s})$

Length of the seasonality $=12$

\section{Summary of Forecasts}

Non-seasonal differentiation of order: 1

Forecasting Model Selected: ARIMA $(2,1,1)$ x $(1,0,1) 12$ with constant

Number of forecasts generated: 96

Number of periods retained for validation: 0

\begin{tabular}{|l|l|l|}
\hline Statistical & $\begin{array}{l}\text { Period of } \\
\text { Estimate }\end{array}$ & $\begin{array}{l}\text { Period of } \\
\text { Validation }\end{array}$ \\
\hline RMSE & 0,785022 & \\
\hline MAE & 0,546065 & \\
\hline MAPE & 19,7006 & \\
\hline I & 0,0984841 & \\
\hline MPE & $-1,66436$ & \\
\hline
\end{tabular}


Summary of ARIMA Model

\begin{tabular}{|l|l|l|l|l|}
\hline Parameter & Dear & Std error. & $T$ & $P$-Value \\
\hline AR(1) & 0,719815 & 0,149729 & 4,80746 & 0,000006 \\
\hline AR(2) & $-0,317099$ & 0,110374 & $-2,87294$ & 0,005072 \\
\hline MA(1) & 0,736021 & 0,127716 & 5,76295 & 0,000000 \\
\hline SAR(1) & 1,32992 & 0,0233672 & 56,914 & 0,000000 \\
\hline SMA(1) & 1,30476 & 0,0367575 & 35,4964 & 0,000000 \\
\hline Media & $-0,103736$ & 0,0662463 & $-1,56592$ & 0,120877 \\
\hline Constant & 0,0204419 & & & \\
\hline
\end{tabular}

Historical Forecast: Yes

Estimated variance of white noise $=0,644428$ with 90 degrees of freedom

Estimated standard deviation of white noise $=0,802763$

Number of Iterations: 17

This procedure predicts future values of monthly inflation \%. The data cover 97 periods of time. Currently, we have identified the model of an autoregressive integrated moving average (ARIMA). This model assumes that the best prognosis available for future data is given by the parametric model that relates the most recent value with the values and previous noise. Each value of monthly inflation \% has been adjusted in the following way, before adjusting the model:

(1) We take simple differences of order 1.

The output summarizes the statistical significance of the terms in the model forecasts. Terms with Pvalues lower than 0.05 were statistically different from zero with a confidence level of $95.0 \%$. The Pvalue for the term $\operatorname{AR}(2)$ is less than 0.05 , which is statistically different from 0 . The P-value for the term MA(1) is less than 0.05 , so that is statistically different from 0 . The P-value for the term SAR(1) is less than 0.05 , so that is statistically different from 0 . The P-value for the term SMA(1) is less than 0.05 , so that is statistically different from 0 . The P-value for the term of the constant is greater than or equal to 0.05 , so that is not statistically significant. You should consider eliminating the term the constant of the model. The estimated standard deviation of the white noise of input is equal to 0,802763 .

This table also summarizes the performance of the model currently selected in adjust historical data. Shown:

(1) the root mean square error (RMSE)

(2) the mean absolute error (MAE)

(3) the percentage of mean absolute error (MAPE) 
(4) the average error (I)

(5) the percentage of average error (MPE)

Each one of the statistics based on the forecast errors are one-forward, which are the differences between the data at time $t$ and the forecasted value at time $t-1$. The first three statistics measure the magnitude of the errors. A better model would give a smaller value. The last two statistical measure the bias. A better model would give a value closest to 0 .
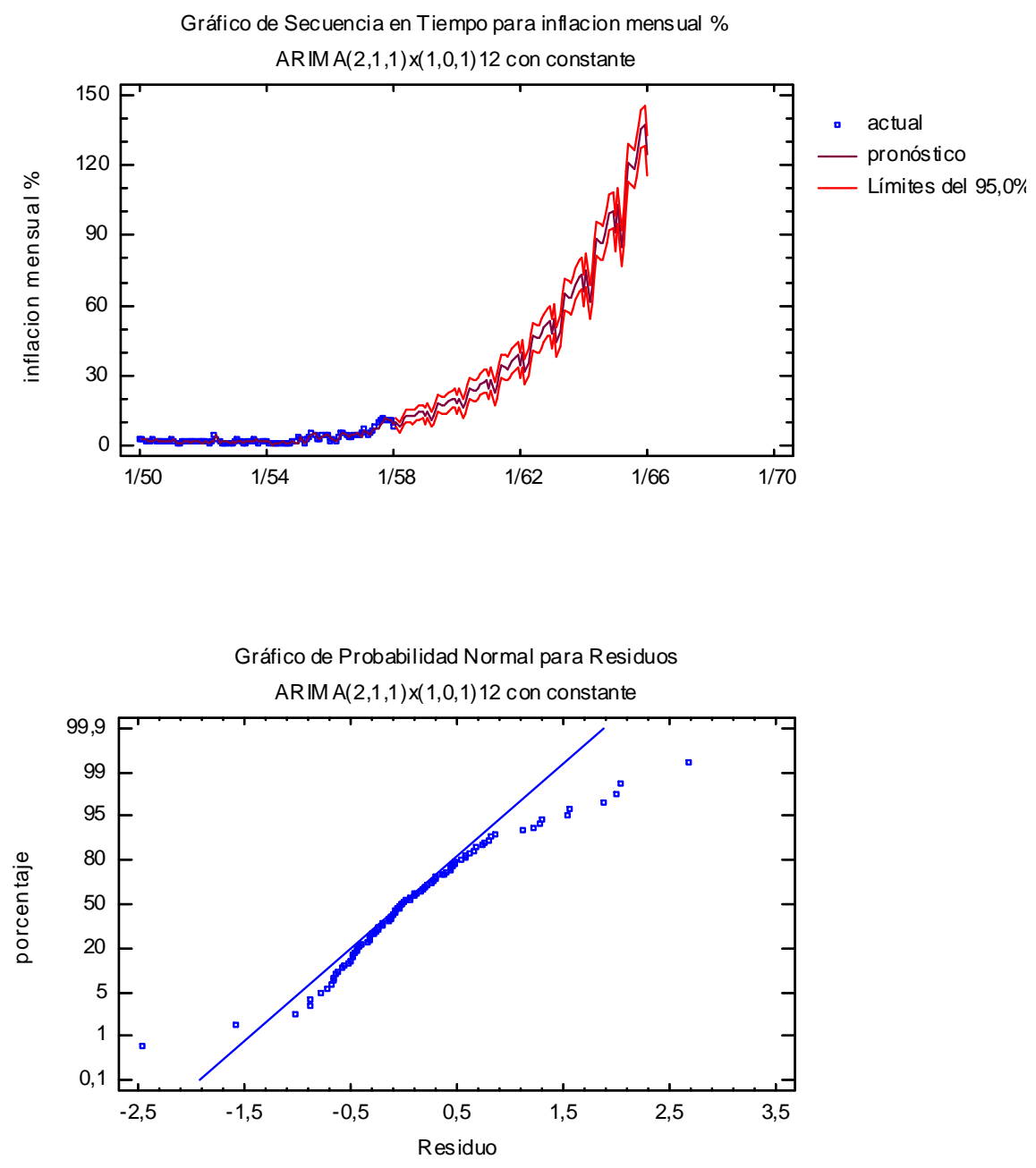


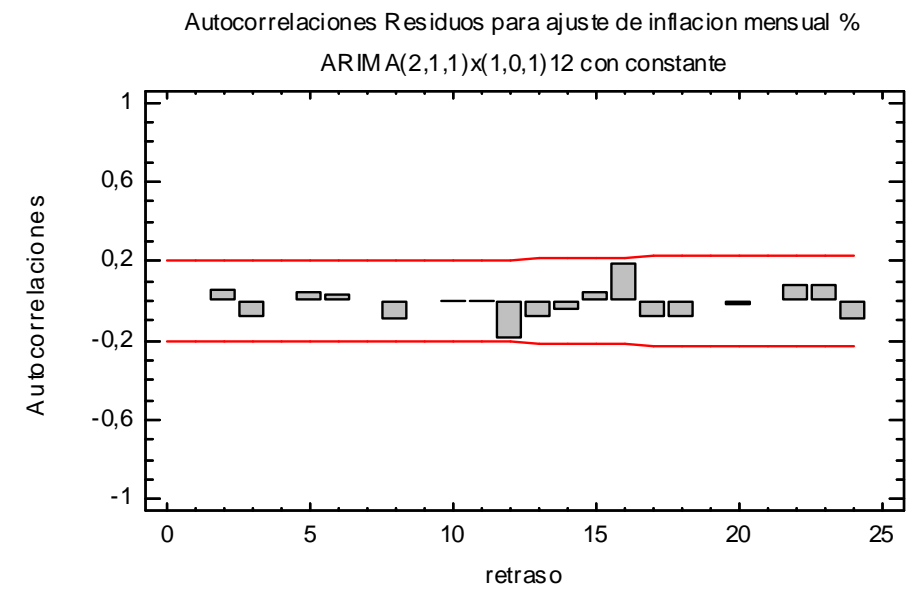

